\title{
Ideias de Van Hiele e Educação Matemática Realística: algumas aproximações
}

\author{
Van Hiele Ideas and the Realistic Mathematical Education: a few \\ approximations
}

\author{
Adriana Quimentão Passos* \\ ORCID iD 0000-0001-5152-2405 \\ Regina Luzia Corio de Buriasco ${ }^{* *}$ \\ ORCID iD 0000-0002-5845-1619 \\ Maria Tereza Carneiro Soares ${ }^{* * *}$ \\ ORCID iD 0000-0003-4645-8124
}

\begin{abstract}
Resumo
Este artigo teve origem em uma pesquisa que investigou possíveis relações entre os princípios de avaliação da Educação Matemática Realística e fases do processo de aprendizagem propostas por Dina Van Hiele-Geldof e Pierre Van Hiele, buscando aproximações. O trabalho foi desenvolvido em uma perspectiva de pesquisa de natureza teórica, do tipo especulativa. Destaca o aspecto didático do trabalho dos Van Hiele, em especial, as fases do processo de aprendizagem: informação, orientação guiada, explicitação, orientação livre e integração. Considera que as fases do processo de aprendizagem dos Van Hiele são mais um elemento auxiliar na elaboração do conhecimento matemático a partir de situações que possam ser matematizadas, desenvolvidas por meio de um processo de reinvenção guiada apoiada em informações coletadas em situações de avaliação.
\end{abstract}

Palavras-chave: Educação Matemática. Educação Matemática Realística. Van Hiele. Princípios de avaliação.

\begin{abstract}
This article originated in a research that investigated possible relations between the principles of Realistic Mathematical Education assessment and the learning process phases proposed by Dina Van Hiele-Geldof and Pierre Van Hiele, seeking approaches. The work was developed in a research perspective of theoretical nature, of the speculative type. It highlights the educational aspect of Van Hiele's work, in particular, the learning process phases: information, guided guidance, exploitation, free orientation, and integration. It considers that the learning

\footnotetext{
* Doutora em Ensino de Ciências e Educação Matemática pela Universidade Estadual de Londrina (UEL). Professora da Secretaria Estadual de Educação do Paraná, Londrina, Paraná, Brasil. Endereço para correspondência: Rua Ernani Lacerda de Athayde, 1200, torre A, apto 705, Gleba Fazenda Palhano, Londina, Paraná, Brasil, CEP: 86.055-630. E-mail: adrianaqpassos@gmail.com.

${ }^{* *}$ Doutora em Educação pela Universidade Estadual Paulista (UNESP). Docente do Departamento de Matemática e do Programa de Pós-Graduação em Ensino de Ciências e Educação Matemática da Universidade Estadual de Londrina (UEL), Londrina, Paraná, Brasil. Endereço para correspondência: Rua Eduardo Benjamin Hosken, 173, apto 501, Centro, Londrina, Paraná, Brasil, CEP: 86020-440. E-mail: reginaburiasco@ gmail.com.

**** Doutora em Educação pela Universidade de São Paulo (USP). Professora associada do Departamento de Planejamento de Administração Escolar da Universidade Federal do Paraná (UFPR), Curitiba, Paraná, Brasil. Endereço para correspondência: UFPR, Setor de Educação, Rua Gal. Carneiro, 460, $5^{\circ}$ andar, CEP 80060-150, Curitiba, Paraná, Brasil. E-mail: mariteufpr@gmail.com.
} 
process phases of Van Hiele's approach are another auxiliary element in the elaboration of mathematical knowledge from situations that can be mathematized, developed through a guided reinvention process based on information collected in assessment situations.

Keywords: Mathematics Education. Realistic Mathematics Education. Van Hiele. Assessment Principles.

\section{Introdução}

A abordagem da Educação Matemática Realística - $\mathrm{RME}^{1}$ considera que a aprendizagem matemática se origina da matematização de situações reais que permitam ao aluno reinventar a matemática mediante a orientação guiada pelo professor. Para a RME, a matemática é o produto final da matematização, o resultado de um processo de organização, esquematização e processamento matemático.

A pesquisa que originou este artigo investigou as possíveis relações entre os princípios de avaliação da Educação Matemática Realística e as fases do processo de aprendizagem propostas por Dina Van Hiele-Geldof (1957) e Pierre Van Hiele (1957, 1986) buscando aproximações.

Foram encontrados indícios da relação entre o trabalho de Van Hiele-Geldof (1957) e Van Hiele (1957) e a Educação Matemática Realística em: Freudenthal (1973, 1991), Treffers e Goffree (1985), Treffers (1987), Van Den Heuvel-Panhuizen (1996). Segundo a autora,

[...] a matematização pode ocorrer em diferentes níveis. Esses níveis de matematização estão conectados aos vários níveis de compreensão através dos quais os alunos podem passar [...]. A essência desse nível teórico, que Freudenthal (1973) empresta das observações e ideias dos Van Hiele, é que a atividade de matematização em um nível mais baixo pode, mais tarde, se tornar um objeto de análise em um nível superior (VAN DEN HEUVEL-PANHUIZEN, 1996, p. 13, tradução nossa).

Freudenthal (1991, p. 96, tradução nossa) também indica que o "que importa no processo de aprendizagem são as descontinuidades [...]. Eu devo a concepção da estrutura de níveis do processo de aprendizagem a minha colaboração com os Van Hiele, um casal que incorporou, por assim dizer, o casamento perfeito de teoria e prática".

A pesquisa que originou este artigo foi desenvolvida na perspectiva da pesquisa de natureza teórica e especulativa em educação, definida como uma obra da produção de enunciados teóricos sobre outros enunciados teóricos (VAN DER MAREN, 1996; MARTINEAU; SIMARD; GAUTHIER, 2001).

Esse tipo de investigação adota três eixos fundamentais: 1) a interpretação, 2) a argumentação e 3) o recontar (MARTINEAU; SIMARD; GAUTHIER, 2001). Nela, o

\footnotetext{
${ }^{1}$ RME é a sigla da expressão inglesa Realistic Mathematics Education.
} 
pesquisador confronta textos de diferentes autores a respeito de um mesmo tema, ou textos do mesmo autor a respeito de um tema escrito em diferentes épocas. A partir da interpretação dos textos, o pesquisador estabelece o referencial teórico e escreve o seu próprio texto, ou seja, uma história, neste caso, acerca das aproximações que foram encontradas entre o trabalho dos Van Hiele e os princípios de avaliação da RME.

\section{Um recontar}

Em 04 de julho de 1957, Dina Van Hiele-Geldof e Pierre Marie Van Hiele concluíram suas respectivas teses de doutorado. Van Hiele defendeu a tese intitulada O problema do insight - uma conexão com a compreensão dos estudantes na aprendizagem da geometria e Van HieleGeldof , A didática da geometria na classe inicial do ensino secundário.

Os trabalhos do casal Van Hiele tiveram uma divulgação relativamente lenta. Na primeira metade da década de 1960, apenas a União Soviética tomou o Modelo de Van Hiele ${ }^{2}$ para desenvolver o novo currículo de Matemática (CROWLEY, 1987; PASTOR, 1993). Esse modelo também foi utilizado na Holanda, durante o desenvolvimento do projeto WISKOBAS (PASTOR, 1993). Segundo Crowley (1987) o Modelo de Van Hiele tornou-se conhecido na América do Norte apenas no início da década de 1970, por meio do livro "Mathematics as an Educational Task" de Hans Freudenthal (1973), e da publicação da conferência de Wirszup, em 1976. Depois disso, o modelo se expandiu para os outros países ocidentais (PASTOR, 1993).

As duas teses se complementam. A de Dina Van Hiele-Geldof (1957) tem um caráter mais prático e a de Pierre Van Hiele (1957), um caráter mais teórico. As teses consistem no desenvolvimento de um modelo teórico envolvendo cinco níveis de compreensão de desenvolvimento do pensamento geométrico. Os trabalhos focam o papel da instrução no ensino de geometria e no papel da instrução para ajudar os estudantes a mudar de um nível para o próximo (FUYS et al. 1984).

A tese de Van Hiele-Geldof (1957) baseou-se em dados coletados por meio de um experimento de ensino, uma situação real de sala de aula desenvolvida em duas turmas de primeiro ano da escola secundária (alunos de doze anos) e teve como objetivo investigar as contribuições da intervenção didática na elaboração do pensamento geométrico, a necessidade

\footnotetext{
2 De acordo com Crowley (1987) o Modelo de Van Hiele consiste em cinco níveis de pensamento denominados: visualização, análise, dedução informal, dedução formal e rigor. Esses níveis descrevem características do processo de pensamento. Segundo esse modelo, a partir da orientação didática, o aluno se move sequencialmente do nível inicial (visualização) para o nível mais alto (rigor). No entanto, poucos estudantes conseguem alcançar o último nível.
} 
do raciocínio lógico na aprendizagem inicial da geometria e o papel da linguagem na transição do pensamento visual para o lógico.

Segundo Van Hiele-Geldof (1957, p. 194), o pensamento dos alunos só se torna um meio de organização da ação didática, quando o professor guia a ação do aluno, tendo em vista os níveis de pensamento e o conteúdo. Em sua tese, encontra-se a reflexão da autora relativa ao papel do professor na transição dos alunos de um nível de pensamento para o subsequente, que, posteriormente, resultou na proposição das fases do processo de aprendizagem e da teoria dos níveis de Van Hiele (1986), conhecida como o Modelo de Van Hiele.

Nessa descrição, também há indícios de aspectos relevantes da Educação Matemática Realística: o papel dos contextos na elaboração do conhecimento matemático; o processo de aprendizagem do professor em relação ao ato de ensinar, que Freudenthal, posteriormente, define como didatização; a descontinuidade no processo de aprendizagem; o papel da linguagem para a elaboração do conhecimento; a relevância dos contextos para a elaboração de conceitos; a unidade dos processos de ensino e de aprendizagem.

"Diz-se que uma criança tem insight em um determinado campo da geometria quando, a partir dos dados e relações geométricas que são fornecidos, é capaz de chegar a uma conclusão em uma situação que nunca tinha enfrentado antes" (VAN HIELE, 1957, p. 1, tradução nossa). De modo geral, considera-se um insight quando o sujeito atua, correta e intencionalmente, frente a uma situação nova.

Para Van Hiele (1957), o insight é essencial para a aprendizagem, por isso é necessário refletir a respeito da organização do ensino. Quais são as condições para se desenvolver o insight? Segundo o autor, para que a didática contribua para um bom funcionamento do insight, existe a necessidade da participação efetiva do aluno nas situações de ensino e de aprendizagem. Para a prática diária em sala de aula, ele sugere que os professores ofereçam ao aluno oportunidades ricas para formar estruturas que podem ser utilizadas em situações-problemas, conforme foi relatado na tese de Dina Van Hiele-Geldof (1957).

Uma questão essencial explorada por Van Hiele (1986) é a sua compreensão de estrutura. Para ele, uma estrutura é reconhecida pelo rigor. A propriedade mais importante de uma estrutura é a possibilidade de ser estendida em função da sua composição. Outra propriedade é ter objetividade. Diferentes pessoas podem continuar uma estrutura da mesma maneira. Ela é um fenômeno que permite que tanto os homens quanto os animais atuem em uma situação que não é exatamente a mesma que eles tinham encontrado antes, eliminando a necessidade de efetuar uma quantidade interminável de tentativas e erros. Elas permitem que as pessoas compreendam umas às outras. $\mathrm{O}$ autor afirma que, quando "uma pessoa que age com 
intenção não age de forma aleatória, ela age de acordo com a estrutura que percebe, o que corresponde à sua estrutura mental, a estrutura de sua expectativa" (VAN HIELE, 1986, p. 24, tradução nossa).

Segundo Van Hiele (1986), a propriedade mais importante dos níveis de pensamento é a descontinuidade, a incoerência entre as suas estruturas. Para demonstrar a existência de incoerências entre os níveis de pensamento, é necessário mostrar as descontinuidades entre um nível e o subsequente.

Para a matemática, mais especificamente para o pensamento geométrico, Van Hiele (1986) lista cinco níveis de pensamento: visual, descritivo, teórico, lógica formal e a natureza das leis lógicas. Segundo o autor, essa classificação é adequada para uma estrutura matemática. Para o processo de aprendizagem, ele ainda acrescenta cinco fases: a da informação, na qual os alunos se familiarizam com o domínio do trabalho; a da orientação guiada, em que os alunos são guiados por tarefas em diferentes relações da rede que deve ser formada; a da explicitação, na qual os alunos se tornam conscientes das relações e procuram expressá-las por meio de palavras, aprendem a linguagem técnica relacionada ao assunto; a da orientação livre, momento em que os alunos aprendem tarefas gerais e encontram seu próprio caminho na rede de relações; a da integração, quando os alunos constroem uma visão geral de tudo o que aprenderam da nova rede de relações.

Van Hiele (1986) observa que, na prática letiva, não é dada muita atenção para os níveis de pensamento. Regularmente, os professores iniciam um conceito a partir de um nível de pensamento que ainda não foi atingido por todos os alunos. Para ele, essa é uma das principais causas das dificuldades de aprendizagem em matemática, pois, quando o professor inicia partindo de um nível que os alunos ainda não dominam, eles tornam-se obrigados a imitar a estrutura da ação do professor. Com isso, em geral, aprendem a reproduzir e não conseguem aplicar seus conhecimentos em uma situação concreta.

A “aprendizagem é a aquisição de novas competências, sob a influência de uma intenção de aprender" (VAN HIELE, 1986, p. 176, tradução nossa). Segundo o autor, durante o processo de aprendizagem, o aluno muda de um nível de conhecimento para o subsequente, e essa mudança é permeada pelas cinco fases do processo de aprendizagem.

De acordo com essas fases, o professor pode orientar o processo de aprendizagem: na fase da informação, oferecendo aos alunos materiais para clarificar o contexto; na fase da orientação guiada, fornecendo material por meio do qual os alunos aprendem as principais ligações no campo de pensamento; na fase da explicitação, oportunizando discussões em classe que irão resultar no uso correto da linguagem; na fase da orientação livre, a partir da oferta de 
materiais, com diferentes possibilidades de uso e de instruções que favoreçam várias performances; na fase da integração, o professor convida os alunos a refletirem a respeito de suas ações.

No livro, Van Hiele (1986) retoma questões tratadas na sua tese e na tese de Van HieleGeldof (1957) que estão relacionadas à RME: o papel da linguagem na elaboração do conhecimento, a orientação guiada, as descontinuidades entre os níveis de conhecimento, o papel dos contextos na elaboração e na avaliação do conhecimento, a fenomenologia didática na mesma perspectiva tratada por Freudenthal, a inversão didática.

No livro "Revisiting Mathematics Education", Freudenthal (1991) trata da ideia de níveis. Para o autor, o que caracteriza os níveis hierárquicos de pensamento, de modo geral, são as operações técnicas que, em um determinado nível, tornam-se base para o pensamento em um próximo nível. A essência da mudança de um nível para o próximo se encontra na palavra reflexão, ou seja, é por meio da reflexão que um assunto de um nível mais baixo se transforma em um nível superior. Freudenthal considerou a reflexão como um princípio essencial para o ensino da matemática na perspectiva da reinvenção guiada.

Para La Bastide-Van Gemert (2006) a teoria de níveis serviu como base, principalmente, para a criação de ideias como inversão antididática e reinvenção guiada. Com o tempo, Freudenthal passou a citar o trabalho dos Van Hiele apenas superficialmente e a considerá-lo somente no aspecto básico, ou seja, construiu a sua própria teoria e não se referiu mais a eles. O trabalho de Freudenthal concentrou-se, então, na luta contra o Movimento da Matemática Moderna.

A abordagem de ensino idealizada por Freudenthal desencadeou pesquisas que foram compondo o corpo teórico da RME, construídas a partir do resultado de projetos, como WISKOBAS e HEWET, desenvolvidos por membros do atual Instituto Freudenthal (I.F.). Essas pesquisas têm uma forte interação teoria e prática, assim como foi feito por Van Hiele-Geldof (1957) e Van Hiele (1957) em suas respectivas pesquisas de doutorado.

O projeto WISKOBAS, por exemplo, contou com a participação de Treffers e de membros do IOWO (Instituto para Desenvolvimento de Educação Matemática), e o projeto HEWET, por membros do I.F., incluindo De Lange, que utilizou dados do projeto para sua tese de doutorado e, posteriormente, desenvolveu a ideia de níveis de competência cognitiva e, por conseguinte, os princípios de avaliação e a Pirâmide de Avaliação. Os dois projetos utilizaram princípios da RME, como a concepção da matemática como uma atividade humana, a matematização, a didatização, a fenomenologia didática, os contextos, o princípio de níveis, a unidade do processo de ensino e de aprendizagem, a avaliação didática. 
O projeto HEWET teve início em 1981, com base em dois pilares: experimentos escolares e formação de professores em serviço. Ele foi conduzido por membros do Research Group on Mathematics Education and Educational Computer Centre (OW \& OC), estabelecido na Universidade de Utrecht. Conforme Verhage e De Lange (1987), o período de desenvolvimento, teste e implantação do currículo da Matemática-A, fruto do projeto HEWET, durou cinco anos.

De Lange (1987) destaca que o programa de Matemática-A deveria ser inspirado pela via da aplicação. Nesse currículo destacava-se o processo de matematização conceitual. A ideia era iniciar o trabalho com os conteúdos de matemática a partir de uma situação problema realística. Essa situação deveria desencadear desequilíbrios cognitivos e a matematização conceitual. Os conteúdos/conceitos abstraídos ou formulados pelos estudantes seriam analisados no contexto do mundo real antes de finalizar o processo da matematização e sistematização do assunto. Segundo De Lange (1987), no currículo da Matemática-A, a matematização sempre caminhou junto com a reflexão, com o insight.

Com relação às considerações teóricas que fundamentam a compreensão do processo de matematização conceitual e, consequentemente, o desenvolvimento dos projetos WISKOBAS e HEWET, De Lange (1987, p. 74, tradução nossa) informa que, quando o projeto de desenvolvimento educacional começou, a "concepção teórica só poderia tornar-se visível por paradigmas de unidades de ensino/aprendizagem. O tempo todo a teoria ainda estava implícita na ação, isto é, na criação da instrução. Gradualmente, a instrução produzida tornou- se uma questão de discussão."

TREFFERS, (1987, p. 242, tradução nossa) afirma que, somente

[...] quando o trabalho terminou, um quadro teórico pôde ser construído, no qual os bem conhecidos níveis de Van Hiele puderam ser adaptados e reinterpretados. [...] A característica do andamento dos trabalhos do WISKOBAS em três fases reflete exatamente o processo de aprendizagem em três níveis, como descrito por Van Hiele.

Durante o desenvolvimento do projeto HEWET, os professores sentiram dificuldade em avaliar o desenvolvimento da atitude crítica, do processo de matematização e do uso de contexto por meio de testes escritos em tempo restrito. Essa dificuldade motivou o grupo a desenvolver outros instrumentos de avaliação, como a prova em duas fases, o teste ensaio, a prova de levar para casa e o teste oral. A intenção era proporcionar aos estudantes oportunidades de mostrar o que sabiam.

Para De Lange (1999), o ensino deveria ter como objetivo a aprendizagem, e as provas e tarefas deveriam ser parte desse processo e não somente uma forma de obter notas. A avaliação deve ter o objetivo de produzir informações que ajudem a melhorar a aprendizagem e a tomada 
de decisões educacionais por todos os envolvidos no processo.

Os instrumentos de avaliação desenvolvidos pelos membros do projeto HEWET foram testados tendo em vista cinco princípios para a avaliação. De acordo com esses princípios, as provas devem melhorar a aprendizagem, permitir que o aluno mostre o que sabe, operacionalizar os objetivos curriculares, não ser mensuradas pela facilidade de acerto e ser inseridas na prática escolar habitual (DE LANGE, 1987).

A intenção com esses princípios é oferecer aos estudantes feedbacks adequados, permitir que eles mostrem o que sabem, favorecer o processo de resolução, dar oportunidade para o estudante explicar o processo de resolução e tomar a avaliação como mais um componente da ação didática. Posteriormente, De Lange (1999) acrescentou outros princípios a essa lista: a matemática deve estar incorporada a problemas realísticos; os alunos devem ter diferentes oportunidades para documentar o que sabem, os critérios de avaliação devem ser públicos, os alunos têm o direito de receber feedback fidedignos a respeito do seu trabalho e uma tarefa de avaliação de qualidade deve ser autêntica e justa. Segundo o autor, esses princípios podem ser tomados como um checklist para os professores que levam a sério a avaliação em sala de aula. Eles explicitam o principal objetivo da Educação Matemática que é tornar os estudantes matematicamente letrados.

De Lange presidiu o Grupo de Especialistas de Matemática ${ }^{3}$ nas avaliações do PISA de 2000, 2003, 2006 e 2009. Por influência do autor, as questões dessa avaliação estavam a serviço de verificar o letramento matemático, que se refere à capacidade de identificar e compreender o papel da Matemática no mundo moderno, de tal forma a fazer julgamentos bem fundamentados, envolver-se com a Matemática e utilizá-la com o objetivo de atender às necessidades do indivíduo no cumprimento de seu papel de cidadão consciente, crítico e construtivo.

Os princípios de avaliação foram levados em consideração por De Lange (1999) para explicitar as competências cognitivas que revelam o letramento matemático. De Lange (1999) sintetizou as competências em três níveis: reprodução, conexão e reflexão. No nível 1 (reprodução), espera-se que o aluno desenvolva tarefas rotineiras. No nível 2 (conexão), esperase que o aluno comece a fazer relações entre os domínios da matemática para a resolução de tarefas. No nível 3 (reflexão), o aluno tem a oportunidade de fazer matemática. Esses três níveis foram representados por De Lange (1999) na Pirâmide de Avaliação (Figura 1), que contempla o conteúdo ou domínio da matemática, os níveis de competência cognitiva e o grau de

\footnotetext{
${ }^{3}$ Chairman of the Mathematics Expert Group of the Organization for Economic Co-Operation And Development - OECD/PISA.
} 
complexidade cognitiva das questões.

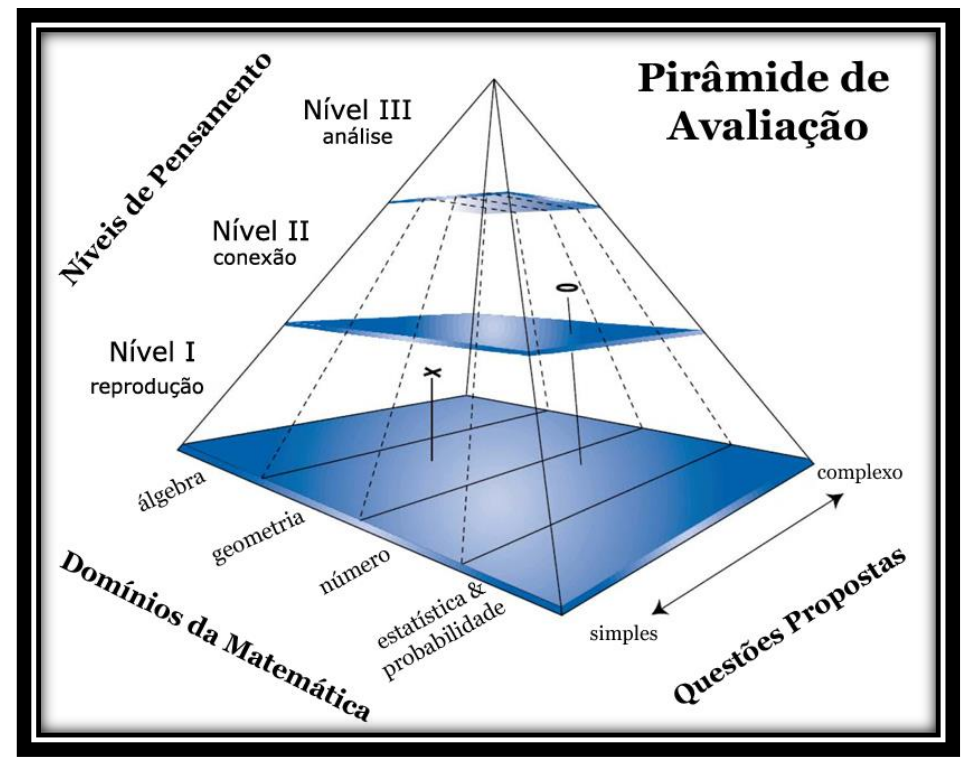

Figura 1 - Pirâmide de Avaliação, De Lange (1999) Fonte: Tradução de Ferreira (2013)

Segundo Van den Heuvel-Panhuizen (1996), a Pirâmide de Avaliação pode servir como uma orientação para elaborar uma prova e para mostrar como as tarefas em uma determinada prova foram distribuídas ao longo dos vários temas, níveis e graus de complexidade cognitiva.

Encontram-se indícios da aproximação entre os trabalhos de Van Hiele-Geldof (1957) e Van Hiele $(1957,1986)$ e a abordagem da Educação Matemática Realística idealizada por Freudenthal (1968, 1973, 1983, 1991) desde a participação deles no Mathematics Study Group of the W.V.O (GOFFREE, 1993). A tese de Van Hiele (1957) foi o primeiro trabalho que Freudenthal orientou na área de ensino da matemática. Ele também participou da elaboração da tese de Van Hiele-Geldof (1957). Segundo ele, o trabalho de Van Hiele-Geldof (1957) era um documento educacional único e valioso.

As teses de Van Hiele-Geldof (1957) e Van Hiele (1957) foram inovadoras para a época, pois tratavam do processo de ensino e aprendizagem da Matemática a partir do relato de situações de sala de aula. Van Hiele-Geldof (1957) investigou as possíveis contribuições da didática para a elaboração do conhecimento e também o papel da linguagem na transição do pensamento visual para o lógico.

Van Hiele (1957) apresentou um estudo a respeito do insight em um contexto didático ${ }^{4}$ destacando o papel do professor. Seu trabalho pauta-se pelos resultados encontrados no estudo de Van Hiele-Geldof (1957), segundo a qual, a linguagem matemática só terá significado para

\footnotetext{
${ }^{4}$ Para o autor, a didática pertence ao mundo das experiências, ele acredita que o estudo do insight deve ser baseado em experiências objetivas, em experiências acessíveis a qualquer investigador sério. A tese dele foi elaborada mediante experiências objetivas.
} 
o aluno se decorrer de uma experiência significativa e se for acompanhada de uma estrutura de linguagem adequada. Recomenda que o professor inicie com a fala cotidiana e, durante o processo de ensino e de aprendizagem, apresente a linguagem matemática. Tanto para Freudenthal $(1973,1991)$ quanto para Van Hiele-Geldof (1957) e Van Hiele (1957, 1986), a linguagem exerce um papel fundamental no processo de elaboração do conhecimento matemático.

Outro ponto de conexão entre Van Hiele-Geldof (1957), Van Hiele $(1957,1986)$ e a abordagem da RME é a compreensão do processo de aprendizagem. Para Freudenthal (1991), a aprendizagem é um processo descontínuo, e o casal Van Hiele compartilha dessa afirmação. Conforme Van Hiele (1957), o processo de aprendizagem segue claramente um curso descontínuo. Cabe ao professor compreender a variação de ritmos e buscar meios para favorecer a mudança de um nível para outro, superior.

Para Freudenthal (1991), os saltos, ou a descontinuidade, revelam a presença de níveis. Durante a elaboração do conhecimento, chega um momento no qual o processo parece parar e, na sequência, ele continua como se o aluno tivesse amadurecido. O professor não consegue explicar o assunto. É como se falasse uma linguagem incompreensível. Conforme Van HieleGeldof (1957) e Van Hiele (1957), o professor deveria estruturar a situação de aprendizagem no conhecimento das relações lógicas entre os níveis em que os alunos se encontram durante a aprendizagem. Quando o aluno atinge um novo nível, ele passa a compreender a linguagem do professor.

Segundo Van Hiele (1986), por vezes, o aluno não compreende a linguagem utilizada pelo professor. É como se eles estivessem em mundos diferentes. Nesse caso, a aprendizagem não ocorre, pois o aluno não consegue estabelecer uma rede de relações. Nessas situações, cabe ao professor guiar o aluno para um nível superior. Para Van Hiele-Geldof (1957) e Van Hiele (1957 e 1986), a transição de um nível para o outro pode ser auxiliada pelo professor por meio da orientação guiada.

Freudenthal (1991) também tratou da orientação guiada utilizando o termo reinvenção guiada, que foi escolhido para explicar como imaginava que a matemática deveria ser aprendida. Para o autor, no contexto do ensino, o que ocorre é uma invenção do conteúdo matemático, por envolver tanto o conteúdo quanto a forma do conhecimento matemático. Ele compreende as invenções como fases do processo de aprendizagem, por isso foi adicionado o prefixo re, de reinvenção, que, mediante o ambiente de instrução do processo de aprendizagem, é acompanhado do adjetivo guiado.

Pode-se inferir que o trabalho de Van Hiele-Geldof (1957) é a raiz do princípio da 
reinvenção guiada. A compreensão de Freudenthal (1991) da matemática como uma atividade humana e da reinvenção guiada é, para a Educação Matemática Realística, o ponto de partida para as aulas de matemática, por meio de um processo de matematização, ou seja, pelo fazer matemática. Dina Van Hiele-Geldof (1957) dá indícios da ideia de matematização ao discorrer a respeito da ordenação de relações para a formação de novas estruturas de pensamento e, também, ao citar que a atividade matemática consiste em esquematizar dados empíricos.

Segundo Treffers (1987), na perspectiva da RME, o significado da reinvenção guiada pode ser sintetizado a) na escolha de situações de aprendizagem a partir da realidade do aluno apropriadas para a matematização horizontal, b) na oferta de ferramentas para a matematização vertical, c) na instrução interativa entre os alunos e entre o professor e os alunos, d) na produção do próprio aluno e, finalmente, e) no entrelaçamento da aprendizagem. Freudenthal (1991) reforça que, nesse processo, o professor deve provocar o pensamento reflexivo, que é um motor forte da invenção matemática.

Por outro lado, Van Hiele (1986) distingue cinco fases na transição de um nível de conhecimento para o nível seguinte: a) informação, na qual os alunos se familiarizam com o conhecimento; b) orientação guiada, em que os alunos são orientados a realizar diferentes tarefas relacionadas à rede de relações que deve ser formada; c) explicitação, em que os alunos tomam consciência das relações e procuram expressá-las verbalmente; d) orientação livre, na qual os alunos aprendem tarefas gerais e e) integração, ou seja, a construção de uma visão geral de tudo que aprenderam.

Tanto as fases estabelecidas por Treffers (1987) para a reinvenção guiada quanto as estabelecidas por Van Hiele (1986) têm a intenção de provocar a reflexão, ou seja, desencadear insight que, na perspectiva de Van Hiele, ocorre quando o aluno atua, correta e intencionalmente, em uma situação nova. Para esse autor, o insight e as situações de aprendizagem estão muito próximos. Após certo tempo em aprendizagem, a pessoa se torna capaz de agir adequadamente diante das situações que surgirem durante o processo de aprendizagem.

A proposta da RME de guiar a ação do aluno por meio da reinvenção guiada provém da ideia da matemática como uma atividade humana, que, na perspectiva de Freudenthal (1973, 1991), significa reconhecê-la como uma atividade da natureza humana. Essa concepção também pode ser reconhecida em Van Hiele-Geldof (1957), ao indicar que a matemática é um conhecimento da mente, assim como do mundo. Ela pode ser vista como um ponto de tangência entre o empírico e o intelectual. Nessa perspectiva, o processo de aprendizagem da matemática ocorre por meio da matematização. 
Conforme La Bastide-Van Gemert (2006), Freudenthal reconheceu que os Van Hiele foram os primeiros a tratarem do processo de matematização, apesar de eles não terem utilizado explicitamente o termo. O real processo de matematização, de produção do conhecimento matemático, ocorre quando o aluno tem a oportunidade de analisar, interpretar, desenvolver seus próprios modelos e estratégias e elaborar argumentos, incluindo provas e generalizações. Esse nível exige habilidades de níveis superiores de pensamento.

A concepção de matematização permeia os projetos desenvolvidos na perspectiva da RME. Por exemplo, no projeto HEWET, desenvolvido por De Lange (1987), a matematização caminha junto com a reflexão, com o insight. Ele propõe iniciar a partir da exploração fenomenológica de um conceito matemático estruturado no primeiro nível, que será processado por meio da reinvenção guiada até alcançar níveis mais elevados, ou seja, um processo de matematização conceitual que é desencadeado a partir de problemas de contexto.

Assim como Van Hiele-Geldof (1957) e Van Hiele (1957) já haviam apontado, De Lange (1987) também indica que os diferentes tipos de contexto interferem no possível processo de matematização e que não é possível avaliar problemas de todos os contextos por meio de instrumentos como a prova escrita em tempo restrito. Existe a necessidade de concepção de diferentes instrumentos de avaliação que permitam ao professor obter informações que possam apoiar o processo de ensino e de aprendizagem. Além disso, também é necessário estabelecer a proporção de questões de cada nível em função do tempo que deve ser destinado a cada tipo de problema.

Um aspecto relevante do trabalho dos Van Hiele, presente nos projetos WISKOBAS e HEWET, é o didático. Nas teses de Van Hiele-Geldof (1957) e Van Hiele (1957) e também no livro "Structure and insight: a theory of Mathematics Education" Van Hiele (1986), os autores sugerem que, antes de estudar um fenômeno, seja feita uma análise do contexto em que esse fenômeno aparece como um símbolo. A perspectiva didática fenomenológica do trabalho deles recebeu forte influência de Langeveld, orientador dela. Dina Van Hiele-Geldof (1957) já indicava que, quando o professor proporciona um contexto apropriado, as crianças podem fazer descobertas.

Outro ponto a ser destacado nos projetos WISKOBAS e HEWET, assim como nas teses dos Van Hiele, é o casamento entre teoria e prática. As pesquisas de Treffers (1987), De Lange (1987), Van Hiele-Geldof (1957) e Van Hiele (1957) partiram do pressuposto da unidade do processo de ensino e de aprendizagem no sentido de considerar que são processos distintos, mas que se complementam, um amálgama. A compreensão da unidade do processo de ensino e de aprendizagem está de acordo com a concepção da RME da matemática como uma atividade 
humana e da avaliação didática, assim como da avaliação como prática de investigação e oportunidade de aprendizagem.

Tendo em vista a perspectiva da avaliação didática, De Lange (1987) partilha da ideia de Van Hiele-Geldof (1957) e Van Hiele (1957) de que o processo de avaliação deve ser coerente com a perspectiva metodológica utilizada em sala de aula, ou seja, não é possível avaliar o insight em matemática, o processo de matematização, a partir de instrumentos de avaliação que retratam apenas um momento, como as provas escritas. Para De Lange (1999), o ensino deve ter como objetivo a aprendizagem, e os testes e tarefas devem fazer parte desse processo. $\mathrm{O}$ objetivo da avaliação deve ser produzir informações que auxiliem o processo de aprendizagem e a tomada de decisões.

O Quadro 1 compila aspectos da reinvenção guiada, nas perspectivas de Santos (2014) e Van Hiele-Geldof (1957).

\begin{tabular}{|l|l|}
\hline $\begin{array}{l}\text { Aspectos da reinvenção guiada para Santos (2014, } \\
\text { p. 38) }\end{array}$ & \multicolumn{1}{|c|}{$\begin{array}{c}\text { Aspectos da reinvenção guiada para Van Hiele- } \\
\text { Geldof (1957) }\end{array}$} \\
\hline $\begin{array}{l}\text { O trabalho em sala de aula tem início com a } \\
\text { proposição de uma situação realística que possibilita } \\
\text { diferentes níveis de matematização. }\end{array}$ & $\begin{array}{l}\text { O professor deve proporcionar aos alunos } \\
\text { experiências a partir das quais pode surgir a } \\
\text { necessidade de ordenação do raciocínio lógico } \\
\text { (matematização). }\end{array}$ \\
\hline $\begin{array}{l}\text { Após resolverem a situação, os alunos podem } \\
\text { interagir uns com os outros e terem a oportunidade de } \\
\text { analisar e discutir estratégias e procedimentos que } \\
\text { utilizaram. }\end{array}$ & $\begin{array}{l}\text { A discussão em classe é umarte essencial } \\
\text { (interação). A partir dela, o professor pode certificar } \\
\text { se as ideias individuais são partilhadas pela classe. }\end{array}$ \\
\hline $\begin{array}{l}\text { Durante e após o trabalho dos alunos, o professor } \\
\text { pode fazer questionamentos para explorar as } \\
\text { resoluções que apresentaram, bem como as } \\
\text { diferenças existentes entre elas, e discutir aspectos } \\
\text { matemáticos subjacentes a essas resoluções } \\
\text { encorajando-os a se interessar por esses aspectos. }\end{array}$ & $\begin{array}{l}\text { O segredo do ensino é a pergunta por quê?. Cabe ao } \\
\text { professor estimular descobertas. }\end{array}$ \\
\hline
\end{tabular}

Quadro 1 - Alguns aspectos da dinâmica da aula sob a perspectiva da reinvenção guiada para Santos (2014) e Van Hiele-Geldof (1957)

Fonte: as autoras

Considerando que cabe ao professor organizar a situação didática, que, na perspectiva da RME, é feita por meio da reinvenção guiada, que tem suas origens no trabalho de Dina Van Hiele-Geldof (1957), espera-se que as tarefas escolhidas atendam aos princípios da avaliação e, na medida do possível, contemplem a Pirâmide de Avaliação.

É na ação didática que se encontram aproximações com o trabalho dos Van Hiele. É nela que o professor, durante o processo de reinvenção guiada, proporciona situações de informação, orientação guiada, explicitação, orientação livre e interação; em que cada aluno irá responder de uma forma e, gradativamente, vai mudando de um nível para o seguinte, por meio de saltos, conforme colocado por Freudenthal, a partir de situações que, preferencialmente, proporcionem matematização. 
Segundo De Lange (1999), o verdadeiro processo de matematização ocorre mediante contextos de terceira ordem no nível de análise (terceiro nível). Van Hiele-Geldof (1957) e Van Hiele (1957) também destacam o papel do contexto para a elaboração do conhecimento matemático. Conforme representado na Pirâmide de Avaliação e, também, na descrição que o autor faz dos níveis, quanto maior a exigência cognitiva mais se reduz a diferença entre os domínios da matemática e o grau de dificuldade. Isso contempla o princípio do entrelaçamento, conforme sintetizado por Ferreira (2013), ao citar Van Den Heuvel-Panhuizen (1996), que indica que domínios como a geometria, os números, as medidas e a manipulação são elementos curriculares fortemente integrados. Além disso, esse entrelaçamento traz coerência para o currículo.

Com relação ao princípio dos níveis, para Van Den Heuvel-Panhuizen (1996) os alunos passam por vários níveis de compreensão. Esses vários níveis ficam bem representados na Pirâmide de Avaliação, na qual a forma tridimensional, representada por três regiões, permite imaginar as questões dispostas dentro dessas regiões. Os níveis de competência cognitiva estabelecidos por De Lange (1999), e representados na Pirâmide, dizem respeito ao tipo de tarefa, ao contexto e à possibilidade de matematização. Por outro lado, os níveis estabelecidos por Van Hiele $(1957,1986)$ estão relacionados às habilidades do aluno em cada um dos níveis. Esses autores olham de modo diferente para os níveis.

\section{Algumas considerações}

Na pesquisa realizada, foram encontrados indícios de princípios da RME em trabalhos dos Van Hiele: a compreensão da matemática como uma atividade humana, o papel do contexto para a elaboração do conhecimento matemático, o processo de matematização, a reinvenção guiada, a unidade do processo de ensino e de aprendizagem, a interação professor e aluno, a necessidade de instrumentos de avaliação adequados para coletar informações que possam colaborar no processo de ensino e de aprendizagem.

Das teses dos Van Hiele e do livro "Structure and insight: a theory of Mathematics Education" (VAN HIELE, 1986), destacam-se as seguintes fases do processo de aprendizagem: informação, orientação guiada, explicitação, orientação livre e integração. Essas fases tratam do aspecto didático da transição entre os níveis de pensamento e colaboram com a concepção do ensino da matemática por meio da reinvenção guiada.

Novas pesquisas poderiam levar em consideração a observação de Van Hiele-Geldof (1957) e Van Hiele (1957) de que o professor deveria estruturar a situação de aprendizagem no 
conhecimento das relações lógicas entre os níveis em que os alunos se encontram durante a aprendizagem. Quando o aluno atinge um novo nível, ele passa a compreender a linguagem do professor, assim como indicado por Freudenthal (1991).

Tomar a avaliação como prática de investigação e oportunidade de aprendizagem implica considerá-la como uma ação didática que deve estar incorporada a todo o processo de ensino e de aprendizagem, de acordo com a perspectiva metodológica adotada pelo professor. $\mathrm{Na}$ abordagem da Educação Matemática Realística, destaca-se o processo de matematização como um meio de favorecer a elaboração do conhecimento matemático, que pode ser conduzido pelo professor por meio da reinvenção guiada. A avaliação, do ponto de vista didático, pode fornecer as informações necessárias para o professor desenvolver as aulas, considerando as fases do processo de aprendizagem diante das descontinuidades ou dos saltos que permeiam a formação de conceitos.

\section{Referências}

CROWLEY, M. L. The Van Hiele model of the development of geomemc thought. In: LINDQUIST, M. M. (Ed.). Learning and teaching geometry, K-12. Reston: National Council of Teachers of Mathematics, 1987. p. 1-16. Yearbook.

DE LANGE, J. Mathematics, insight and meaning. Utrecht: Ed. OW and OC, 1987.

DE LANGE, J. Framework for classroom assessment in mathematics. 1999. Disponível em: $<$ http://www.fi.uu.nl/catch/products/framework/de_lange_frameworkfinal.pdf $>$. Acesso em: $12 \mathrm{jan}$. 2009.

FERREIRA, P. E. A. Enunciados de Tarefas de Matemática: um estudo sob a perspectiva da Educação Matemática Realística. 2013. 121f. Tese (Programa de Pós-Graduação em Ensino de Ciências e Educação Matemática) - Universidade Estadual de Londrina, Londrina, 2013.

FREUDENTHAL, H.. Why to teach mathematics so as to be useful. Educational Studies in Mathematics, Dordrecht, v. 1, n. 1-2, p. 3-8, 1968.

FREUDENTHAL, H. Mathematics as an educational task. Dordrecht: D. Reidel Publishing, 1973.

FREUDENTHAL, H. Didactical phenomenology of mathematical structures. Dordrecht: D. Reidel Publishing, 1983.

FREUDENTHAL, H. Revisiting mathematics education. Dordrecht: Kluwer Academic Publishers, 1991.

FUYS, D. et al. English translation of selected writings of Dina van Hiele-Geldof and Pierre $M$. van Hiele. 1984. Disponível em: <http://eric.ed.gov/?id=ED287697>. Acesso em: 14 ago. 2014.

GOFFREE, F. HF: working on mathematics education. Educational Studies in Mathematics, Dordrecht, v. 25, n. 1-2, p. 21-49, 1993.

LA BASTIDE-VAN GEMERT, S. "Elke positieve actie begint met critiek": Hans Freudenthal en de 
didactiek van de wiskunde. Hilversum: Uitgeverij Verloren, 2006.

MARTINEAU, S.; SIMARD, D.; GAUTHIER, C. Recherches théoriques et spéculatives:

considérations méthodologiques et épistémologiques. Recherches Qualitatives, Montreal, v. 22, n. 3, p. 32, 2001.

PASTOR, A. J. Aportaciones a la interpretación y aplicación del modelo de Van Hiele: la enseñanza de las isometrias del plano. La evoluación del nível de razonamiento. 1993. 379 f. Tese (Doctoral en Didàctica de la Matemática) - Universitat de Valência, Valência, 1993.

SANTOS, E. R. dos. Análise da produção escrita em matemática: de estratégia de avaliação a estratégia de ensino. 2014. 158 f. Tese (Doutorado em Ensino de Ciências e Educação Matemática) Universidade Estadual de Londrina, Londrina. 2014.

TREFFERS, A.; GOFFREE, F. Rational analysis of realistic mathematics education. In: STREEFLAND, L. (Ed.). Proceedings of the ninth International Conference for the Psychology of Mathematics Education. Utrecht: University of Utrecht, 1985. v. 2, p. 97-123.

TREFFERS, A. Three dimensions: a model of goal and theory description in mathematics instruction: the Wiskobas project. Dordrecht: D. Reidel Publishing, 1987.

VAN DEN HEUVEL-PANHUIZEN, M. Assessment and realistic mathematics education. Utrecht: Ed. CD-ß Press, Freudenthal Institute, Utrecht University, 1996.

VAN DER MAREN, J.-M. Méthodes de recherche pour l'éducation. Bruxelles: De Boeck and Larcier, 1996.

VAN HIELE-GELDOF, D. The didactics of geometry in the lowest class of secondary school. 1984. 206 p. Tesis (Doctoral in Mathematics and Natural Sciences) - Universidad de Utrecht, Utrecht, Traducción al inglés en Fuys, 1957.

VAN HIELE, P. M. El problema de la comprensión (en conexión con la comprensión de los escolares en el aprendizaje de la geometría). 1957. $151 \mathrm{f}$. Tese (Doctoral in Matemáticas y Ciencias Naturales) - Universidad de Utrecht, Utrecht. Traducción al español para el proyecto de investigación Gutiérrez y otros, 1991. No publicada.

VAN HIELE, P. M. Structure and insight: a theory of mathematics education. New York: Academic Press, 1986.

VERHAGE, H. B.; DE LANGE, J. The HEWET project. In: BERGERON, J. C. (Ed.). Proceedings of the eleventh International Conference on the Psychology of Mathematics Education. Montreal: Eric, 1987. v. 3, p. 249-254.

Submetido em 05 de Janeiro de 2019. Aprovado em 31 de Julho de 2019. 